\section{CONHECIMENTO E ACESSO DE IDOSOS AO SERVIÇO DE GERIATRIA DE MOGI DAS CRUZES/SP}

\author{
Older people's knowledge about and access to the geriatrics \\ service of Mogi das Cruzes, São Paulo
}
Conocimiento y acceso de mayores a los servicios de geriatría de Mogi das Cruzes/SP

\section{RESUMO}

Objetivo: Avaliar o conhecimento e o acesso de idosos ao serviço de geriatria de uma policlínica localizada em Mogi das Cruzes, SP. Métodos: Participaram 200 idosos que aguardavam uma consulta ambulatorial na sala de espera da Policlínica da Universidade de Mogi das Cruzes. Esses idosos responderam a um questionário no período de setembro de 2013 a janeiro de 2014. Resultados: Dos avaliados, 58\% $(n=116)$ dos participantes afirmaram saber da existência do médico geriatra, porém, apenas $9,5 \%(n=19)$ foram atendidos por esse especialista. Quanto aos idosos que costumam passar apenas por uma única especialidade para consultas rotineiras, $8 \%(\mathrm{n}=16)$ buscam especialidades diferentes das de clínico geral, cardiologista e ginecologista. Dentre essas outras especialidades, apenas $17,6 \%(n=35)$ buscam o geriatra. A maioria dos participantes, 99,5\% $(n=199)$, considera importante que o idoso passe por um médico geriatra. Conclusão: A maioria dos idosos indagados conhece a especialidade geriátrica, porém nunca tiveram a oportunidade de se consultar com um médico geriatra, embora reconheçam que isso devesse ocorrer. Apesar da existência desta especialidade na policlínica, pode-se dizer que a população não está informada sobre a disponibilidade dos atendimentos desse profissional no serviço municipal.

Descritores: Envelhecimento; Geriatria; Serviços de Saúde para Idosos.

\section{ABSTRACT}

Objective: To assess older people's knowledge about and access to the geriatrics service of a polyclinic located in Mogi das Cruzes, São Paulo. Methods: Participants were 200 older people waiting for an outpatient consultation in the waiting room of the Polyclinic of the University of Mogi das Cruzes. These older people answered a questionnaire in the period from September 2013 to January 2014. Results: 58\% (n=116) of participants reported knowing about the existence of a geriatric physician, but only 9,5\% $(n=19)$ were served by this specialist. Regarding older people who are usually served by one single medical specialty in routine consultations, $8 \%(n=16)$ sought specialists other than general practitioner, cardiologist and gynecologist. As for these other specialties, only $17.6 \%(n=35)$ sought the geriatrician. Most participants $(99.5 \%, n=199)$ think it is important that older people are seen by a geriatric physician. Conclusion: Most older interviewees know the geriatric specialty; however, they never had the opportunity to consult a geriatric physician even though they recognize that this should occur. Despite the existence of this specialty in the polyclinic, it can be said that the population is not informed about the availability of consultations with such professional in the municipal service.

Descriptors: Aging; Geriatrics; Health Services for the Aged.
Artigo Original

\author{
Angela Alves Correia de Souza ${ }^{(1)}$ \\ Karis de Campos ${ }^{(2)}$ \\ Ivone Panhoca ${ }^{(3)}$
}

1) Universidade de Mogi das Cruzes - UMC - Mogi das Cruzes (SP) - Brasil

2) Universidade de Mogi das Cruzes - UMC - Guarulhos (SP) - Brasil

3) Universidade de Mogi das Cruzes - UMC - São Paulo (SP) - Brasil

Recebido em: 27/04/2016

Revisado em: 05/09/2016

Aceito em: 11/10/2016 


\section{RESUMEN}

Objetivo: Evaluar el conocimiento y el acceso de mayores al servicio de geriatría de una policlínica localizada en Mogi das Cruzes, SP. Métodos: Participaron 200 mayores que esperaban por la consulta de ambulatorio en la sala de espera de la Policlinica de la Universidad de Mogi das Cruzes. Esos mayores contestaron a una encuesta entre septiembre de 2013 y enero de 2014. Resultados: De los evaluados, el 58\% (n=116) de los participantes afirmaron saber que habia el médico geriatra pero el 9,5\% (n=19) fueron asistidos por ese especialista. Respecto a los mayores que suelen pasar solamente por una única especialidad para las consultas de rutina, el $8 \%(n=16)$ buscan especialidades distintas de las del clínico general, del cardiólogo y del ginecólogo. De entre esas otras especialidades solamente el 17,6\% $(n=35)$ procuran el geriatra. La mayoría de los participantes, el 99,5\% $(n=199)$ consideran importante que el mayor pase por un médico geriatra. Conclusión: La mayoría de los mayores investigados conoce la especialidad geriátrica pero nunca tuvieron la oportunidad de una consulta con un medico geriatra aunque reconocen que eso debería ocurrir. A pesar de la existencia de esta especialidad en la policlínica, se puede decir que la población no tiene la información de que hay disponibilidad de atendimientos de parte de este profesional en el servicio municipal.

Descriptores: Envejecimiento; Geriatría; Servicios de Salud para Ancianos.

\section{INTRODUÇÃO}

Verifica-se, nos dias de hoje, um acelerado crescimento da população idosa devido ao aumento da expectativa de vida e um decaimento da taxa de fecundidade. Embora esse aumento de pessoas com idade superior a 60 anos possa ser um bom indicativo da eficácia das políticas públicas, ao mesmo tempo, ele remete a sociedade à reflexão de se adaptar às necessidades dessa nova faixa etária ${ }^{(1)}$.

A estimativa é que a população mundial com 60 anos ou mais duplique até 2050, demandando modificações sociais, inclusive nos serviços de saúde ${ }^{(2)}$. Segundo a Fundação de População das Nações Unidas (UNFPA), a metade dessa população estará vivendo em países em desenvolvimento, como o Brasil ${ }^{(3)}$.

O Censo de 2010 ratificou a grande modificação na pirâmide etária brasileira, apresentado uma reduzida quantidade de crianças de até 5 anos e um crescente número de pessoas acima dos $60 \operatorname{anos}^{(4)}$. É fundamental, então, que haja investimentos na saúde, com capacitação de profissionais para atuar nessa área ${ }^{(5-7)}$. Nesse sentido, o Estatuto do Idoso (2003), dispõe a respeito da necessidade de se inserir as disciplinas de gerontologia e geriatria nos currículos de graduação ${ }^{(8)}$ Isso também aponta a necessidade de investimento, independentemente dos custos, para que as demandas sejam assistidas ${ }^{(9)}$ Há extrema relevância em que os profissionais também recebam uma formação humana, pois a velhice, o sofrimento e a morte têm perdido o seu impacto de sensibilização ${ }^{(10)}$.

O geriatra é o profissional médico especialista responsável tanto pela promoção de saúde quanto pelo tratamento de doenças e incapacidades da velhice ${ }^{(11)}$. Dessa forma, o atendimento do idoso deve ser de forma holística, considerando-o integrado em um meio social, familiar, político e histórico ${ }^{(12)}$. O médico geriatra não irá apenas se ater às intervenções no âmbito de atenção secundária, mas também no de promoção de saúde e prevenção de doenças, ainda na atenção primária, orientando, por exemplo, sobre a imunização por meio de vacinas, sobre as mudanças no estilo de vida e fazendo aconselhamento ${ }^{(13)}$, pois as necessidades existentes nessa faixa etária não são resolvidas em curto ou médio prazo, e devem ser assistidas permanentemente ${ }^{(6)}$.

No que diz respeito à região do Alto Tietê, de acordo com o Instituto Brasileiro de Geografia e Estatísticas (IBGE), em 2010, havia em Mogi das Cruzes 39,816 mil idosos, o que representa $10,1 \%$ da população da cidade. A perspectiva do IBGE é de que o número de idosos triplique até $2050^{(14)}$.

Segundo investigações, dos 10 municípios que compõem na região do Alto Tietê, apenas Mogi das Cruzes e Poá oferecem a especialidade de geriatria pela rede municipal de saúde. As averiguações atestam o número insuficiente de profissionais geriatras na região de todo Alto Tietê frente à demanda existente ${ }^{(15)}$.

Dessa forma, esse trabalho objetivou avaliar o conhecimento e o acesso de idosos ao serviço de geriatria de uma policlínica localizada em Mogi das Cruzes, São Paulo.

\section{MÉTODOS}

Trata-se de uma pesquisa do tipo quantitativa e transversal. A amostra da pesquisa se deu por conveniência e participaram 200 idosos, com idade de 60 anos ou mais, que estavam sendo atendidos na Policlínica da Universidade de Mogi das Cruzes, SP, no período de setembro de 2013 a janeiro de 2014. Os idosos foram abordados para participar dessa pesquisa enquanto aguardavam as consultas médicas nas salas de espera das diversas especialidades ambulatoriais desta policlínica, no período da manhã e da tarde. Enfatizase que a policlínica não atende apenas cidadãos da cidade de Mogi das Cruzes, mas também de cidades vizinhas inseridas na região do Alto Tietê.

Adotou-se como critérios de exclusão dos participantes a serem convidados para compor a pesquisa a presença 
de deficiências significativas, como comprometimento das funções cognitivas ou auditivas sem intervenção, dificuldade de compreensão, incapacidade de assinar o nome e a ausência de acompanhante.

Os idosos que aceitaram participar desse estudo responderam um questionário com 8 questões objetivas, contendo dados de identificação, idade, sexo, estado civil, conhecimento da especialidade geriatria, consulta com esse especialista e a opinião sobre essa especialidade. Houve uma padronização entre ambas as pesquisadoras de forma que elas fizessem a entrevista da mesma maneira, adotando os mesmos métodos. O questionário foi lido para os pacientes, explicado quando necessário e a própria pesquisadora anotava as respostas.

Utilizou-se para tabulação dos dados o software BioEstat versão 5.0 e a expressão se deu através de frequências simples e percentual. Os testes de Quiquadrado $\left(\chi^{2}\right)$ e Mann-Whitney foram aplicados para avaliar as diferenças encontradas, adotando-se $p<0,05$ como nível de significância além de estatística descritiva para tabulação dos dados.

O presente trabalho foi submetido ao parecer do Comitê de Ética e Pesquisa da Universidade de Mogi das Cruzes (UMC), cuja resposta foi favorável sob parecer de $\mathrm{n}^{\mathrm{0}}$ 348.408. Todos os participantes desta pesquisa assinaram o Termo de Consentimento Livre e Esclarecido (TCLE). Dessa forma, cumpriram-se todos os preceitos éticos da Resolução nº. 466/2012.

\section{RESULTADOS}

O grupo foi constituído de 81 homens (40,5\%) e 119 mulheres $(59,5 \%)$, com idade igual ou maior que 60 anos. A idade mínima foi de 60 e a máxima de 88 anos, com desvio padrão de 7,1 anos. O valor da mediana foi de 68 anos, sendo 70 anos a média de idade dos participantes. Quanto ao estado civil, a maioria $(58 \%, \mathrm{n}=116)$ revelou ser casado, $8 \%(\mathrm{n}=16)$ solteiro, $7 \%(\mathrm{n}=14)$ divorciado, $1 \%(\mathrm{n}=2)$ união estável e $26 \%(n=52)$ com outras situações maritais.

No que diz respeito à pergunta sobre o conhecimento da existência do médico geriatra, $58 \% \quad(n=116)$ dos participantes afirmaram saber da existência do médico especialista em idosos (Tabela I). Porém, 90,5\% ( $\mathrm{n}=181)$ dos participantes relataram nunca ter sido atendido por esse tipo de médico.

Quando se analisou a correlação entre idade e o conhecimento sobre a especialidade geriatria, verificouse não haver tendência de maior conhecimento sobre a especialidade com o aumento da idade. Utilizou-se nível de decisão igual a 0.05 (Tabela II). Dentro desse mesmo contexto, observou-se não haver tendência de maior procura pelo geriatra com o aumento da idade (Tabela II).

Dos 9,5\% $(\mathrm{n}=19)$ atendidos pelo geriatra, $80 \%(\mathrm{n}=15)$ relataram ter notado diferença entre o atendimento do médico geriatra e o atendimento do médico pelo qual costumavam passar rotineiramente antes de se consultarem com o geriatra. Dentre as diferenças relatadas, destacam-se como

Tabela I - Conhecimento e acesso ao serviço de geriatria. Mogi das Cruzes, São Paulo, 2015.

\begin{tabular}{lrrrrrr}
\hline \multirow{2}{*}{ Variáveis } & \multicolumn{2}{c}{ Sim } & \multicolumn{2}{c}{ Não } & \multicolumn{2}{c}{ Total } \\
\cline { 2 - 7 } & n & \% & n & \% & n & \% \\
\hline Conhecimento sobre existência do médico Geriatra & 116 & $58 \%$ & 84 & $42 \%$ & 200 & $100 \%$ \\
Quantidade de idosos atendidos por um médico Geriatra & 19 & $9,5 \%$ & 181 & $90,5 \%$ & 200 & $100 \%$ \\
\hline
\end{tabular}

Tabela II - Respostas dos idosos com relação ao conhecimento e ao atendimento por um geriatra. Mogi das Cruzes, São Paulo, 2015.

\begin{tabular}{lccccc}
\hline \multirow{2}{*}{ Variáveis } & \multicolumn{5}{c}{ Idade } \\
\cline { 2 - 5 } & $\mathbf{6 0 - 6 5}$ & $\mathbf{6 6 - 7 0}$ & $\mathbf{7 1 - 7 5}$ & $\mathbf{7 6 - 7 9}$ & $\geq \mathbf{8 0}$ \\
\hline Conhecimento sobre geriatria & 42 & 29 & 20 & 14 & 12 \\
$\quad$ Sim & 24 & 22 & 16 & 9 & 12 \\
Não & & & & & \\
Atendimento por geriatra & 7 & 2 & 4 & 3 & 3 \\
Sim & 59 & 49 & 32 & 20 & 21 \\
Não & & & & & \\
\hline
\end{tabular}


as mais citadas: consulta mais longa, maior entendimento das problemáticas da idade, atendimento mais específico e detalhado, além de mais atenção do médico durante a consulta. Dos participantes atendidos pelo médico geriatra, $55 \%(\mathrm{n}=110)$ mencionaram o local onde a consulta foi realizada sendo que, desse total, $54,5 \%(n=109)$ dos atendimentos concentrou-se no município de Mogi das Cruzes.

Em relação à questão sobre com que tipo de médico esses participantes costumam se consultar rotineiramente, $59,5 \%(\mathrm{n}=119)$ assinalaram apenas um tipo de médico, $29 \%(n=58)$ passam por duas especialidades para consultas de rotina e $11,5 \%(n=23)$ costumam passar por três especialidades ou mais.

No que se refere aos idosos que habitualmente passam apenas por uma única especialidade, $40 \%(\mathrm{n}=80)$ se dirigem ao clínico geral, $9,5 \%(\mathrm{n}=19)$ procuram o cardiologista, $2 \%$ $(n=4)$ recorrem ao ginecologista e $8 \%(n=16)$ buscam outras especialidades. Dentre essas outras especialidades, $29,4 \%$ $(n=59)$ vão ao oftalmologista e $17,6 \%(n=35)$ buscam o geriatra.

Daqueles que buscam por dois especialistas em consultas de rotina, a maioria $(15,5 \%, \mathrm{n}=31)$ relatou ter ido ao clínico geral e ao ginecologista, sendo que 3,4\% ( $n=7)$ recorrem ao cardiologista e geriatra.

Por fim, quando os participantes foram indagados se consideravam importante que o idoso passasse por um médico específico, o médico geriatra, 99,5\% ( $\mathrm{n}=199)$ referiram que sim. Tal dado demonstra a percepção que eles têm sobre a importância do acompanhamento e intervenção do médico geriatra na saúde do idoso.

\section{DISCUSSÃO}

Dos 200 idosos atendidos na Policlínica da Universidade de Mogi das Cruzes, verificou-se que 59,5\% da amostra era do sexo feminino. A maioria da amostra (58\%) revelou ser casada, e a média de idade dos participantes foi de 70 anos.

$\mathrm{O}$ fato de a amostra ter sido composta prioritariamente de mulheres vai ao encontro do levantamento feito pelo IBGE no Censo de $2010^{(4)}$, em que a faixa etária que abrange a terceira idade, a saber, 60 anos ou mais, é também composta por um número superior de mulheres em detrimento da quantidade de homens, atestando para uma longevidade maior do sexo feminino que do sexo masculino, fato também observado por outros autores ${ }^{(14,16)}$. Um estudo sugeriu que essa diferença no número feminino em relação ao masculino pode ser ocasionada pela maior procura de atendimento médico por mulheres ${ }^{(17)}$.

Em outros estudos também se constatou que a maioria dos participantes eram casados ${ }^{(18,19)}$. Em um deles, a média de idade dos participantes foi de $69,1 \operatorname{anos}^{(19)}$. Tais achados foram semelhantes aos encontrados no presente trabalho. O fato de se ter uma média de idade em torno dos 70 anos demonstra o real aumento da expectativa de vida observado pelo IBGE $^{(4)}$, já que, no Censo de 1990, a esperança de vida era de 67,7 anos.

No que diz respeito à pergunta do questionário em que se indaga o conhecimento sobre a existência do médico geriatra, $58 \%$ dos participantes afirmaram saber da existência do médico especialista em idosos (Tabela I). Porém, 90,5\% dos entrevistados relataram nunca terem sido atendidos por esse tipo de médico, o que corrobora a realidade brasileira de que, no ano de 2013, havia 1.149 titulados em geriatria, o que corresponde a $0,43 \%$ de todos os médicos especialistas no Brasil. Importante mencionar que existem, no Brasil, 0,59 geriatras para cada 100.000 habitantes ${ }^{(20)}$. A realidade da região do Alto Tietê não é diferente, havendo uma deficiência de geriatras frente à demanda apresentada, como demonstrado ${ }^{(15)}$.

A maioria dos indivíduos atendidos por um geriatra (80\%) mencionou ter notado diferença entre o atendimento do médico geriatra e o atendimento do médico pelo qual costumava passar rotineiramente antes de se consultar com o geriatra, pontuando características como: consulta mais longa, maior entendimento das problemáticas da idade, atendimento mais específico e detalhado, além de mais atenção do médico durante a consulta. Esse dado vem ao encontro de uma pesquisa realizada em Kentucky (EUA), em que se identificou a necessidade de conhecimento e habilidades específicas no tratamento de idosos durante as consultas, sendo perceptível que as competências geriátricas são diferentes das competências generalistas ${ }^{(9)}$. Isso compactua com o diferencial da abordagem geriátrica, que é ampla para avaliação clínica e inclui aspectos psicossociais, sendo, portanto, uma consulta mais prolongada segundo informações da Sociedade Brasileira de Geriatria e Gerontologia ${ }^{(21)}$.

Dos participantes que foram atendidos pelo médico geriatra, $55 \%$ mencionaram o local onde a consulta foi realizada, sendo que, desse total, $54,5 \%$ foram atendidos no município de Mogi das Cruzes. Esse dado é condizente com a investigação feita no Alto Tietê, em que é relatado que apenas os municípios de Mogi das Cruzes e Poá dispõem de especialidade geriátrica em sua rede municipal de saúde ${ }^{(15)}$. Ressalta-se que a Policlínica da Universidade de Mogi das Cruzes, local onde a pesquisa foi coletada, atende a população mogiana e também das cidades circunvizinhas, portanto, alguns participantes eram do município de Mogi das Cruzes e outros, não.

Em um estudo realizado em Campinas(22), foram entrevistados idosos que frequentavam ambulatórios e foi observado que maioria da amostra seria atendida no 
departamento de medicina interna, seguido pelo setor de oftalmologia e, em terceiro lugar, no de cardiologia, corroborando o achado deste estudo, em que a maioria dos sujeitos passam pelo clínico geral (40\%), seguido pelo cardiologista $(9,5 \%)$, sendo a especialidade de oftalmologia a mais procurada dentre os $8 \%$ que procuram outras especialidades, excluindo-se clínico geral, cardiologista e ginecologia nesta população $(29,4 \%)$.

A grande procura pelo médico clínico geral possivelmente se dá pelo fato deste ser o primeiro a fornecer atendimento em Unidades Básicas de Saúde, local procurado pela grande maioria dos idosos. Quanto à procura de cardiologista, esta se justifica pelo fato de haver maior incidência das doenças cardiovasculares à medida que o indivíduo vai envelhecendo, o que compromete sua qualidade de vida e expõe uma relevante causa de morbidade e mortalidade ${ }^{(23)}$. Outro fato que chama atenção é a falta de um médico de referência e que muitos idosos passam por mais de um médico especialista, demonstrando a carência de programas de atenção à saúde do idoso ${ }^{(24)}$

A carência de médicos geriatras nos serviços de saúde é evidenciada em estudo feito em Unidades Básicas de Saúde de Cascavel/ $\mathrm{PR}^{(25)}$ e em estudo realizado no Ceará, nas Redes de Atenção à Saúde de Fortaleza ${ }^{(26)}$, mostrando, portanto, uma demanda muito maior que a oferta. Esse dado corrobora o achado na presente pesquisa, uma vez que apenas $10 \%$ da amostra já foi atendida por um médico geriatra. Com relação aos $8 \%$ que procuram outra especialidade médica, apenas $17,6 \%$ recorrem ao médico geriatra para suas consultas de rotina; e somente $3,44 \%$ dos idosos buscam esta especialidade juntamente com a especialidade de cardiologia para consultas rotineiras.

Por fim, quando os participantes da coleta foram indagados se consideravam importante que o idoso passasse por um médico específico, o médico geriatra, 99,5\% referiram que sim. Tal dado demonstra a percepção que eles têm sobre a importância do acompanhamento e intervenção do médico geriatra na saúde do idoso.

\section{CONCLUSÃO}

Assim, conclui-se que a maioria dos idosos indagados conhece a especialidade geriátrica, porém, a maioria nunca teve a oportunidade de se consultar com um médico geriatra, embora também reconheça que isso devesse ocorrer.

Apesar do serviço municipal ofertar o profissional médico geriatra, mesmo que em pequeno número, pode-se dizer que a população não está informada sobre a existência dele, havendo necessidade de uma maior divulgação entre os pacientes idosos.

\section{AGRADECIMENTOS}

Ao Conselho Nacional de Desenvolvimento Científico e Tecnológico (CNPq/PIBIC) pelo auxílio financeiro e ao Dr. Melquiades Machado Portela, diretor da Policlínica da Universidade de Mogi das Cruzes (UMC), por sua prestatividade e solicitude.

\section{REFERÊNCIAS}

1. World Health Organization. World Health Statistics. In: Life expectancy and mortality: parte III. France: WHO; 2012. p. 51-61.

2. World Health Organization. World report on ageing and health [Internet]. Luxembourg: WHO; 2015 [acesso em 2016 Set 7] Disponível em: http://apps.who.int/ iris/bitstream/10665/186463/1/9789240694811_eng. pdf?ua=1

3. Fundo de População das Nações Unidas. Envelhecimento do século XXI: celebração e desafio - resumo executivo [Internet]. Nova Iorque: UNFPA; 2012 [acesso em 2016 Set 7]. Disponível em: https:// www.unfpa.org/sites/default/files/pub-pdf/PortugueseExec-Summary_0.pdf

4. Instituto Brasileiro de Geografia e Estatística. Primeiros resultados definitivos do Censo 2010 [acesso em 2013 Mar 29]. Disponível em: http://www.ibge.gov.br/ home/presidencia/noticias/noticia_visualiza.php?id noticia $=1866 \&$ id_pagina $=1 \&$ titulo $=$ Primeirosresultados-definitivos-do-Censo-2010:-populacao-doBrasil-e-de-190.755.799-pessoas

5. Galera SC, Almeida MM, Gabrielle RR, Aragão LP, Freitas LRS, Freire JB Neto. Estágio supervisionado em Atenção à Saúde do Idoso do Curso de Medicina da Universidade de Fortaleza. ABMES Cad. 2011;21: $35-45$

6. Brasil VJW, Batista NA. O ensino de geriatria e gerontologia na graduação médica. Rev Bras de Ed. Méd. 2015;39(3):344-51.

7. Costa NRCD, Aguiar MIF, Rolim ILTP, Rabelo PPC, Oliveira DLA, Barbosa YC. Política de saúde do idoso: percepção dos profissionais sobre sua implementação na atenção básica. Rev Pesquisa Saúde. 2016;16(2):95101.

8. Câmara dos Deputados (BR). Legislação sobre o idoso. Brasília; 2003 [acesso em 2013 Mar 29]. Disponível em: http://www2.camara.leg.br/responsabilidadesocial/acessibilidade/legislacao-pdf/Legislaoidoso.pdf 
9. Bardach SH, Rowles GD. Geriatric education in the health professions: are we making progress? Gerontologist. 2012;52(5):607-18.

10. Caretta F. Geriatrics education and health professions today: responding to critical need. PAJAR. 2015;3(1): 2-7.

11. Morley JE. A brief history of geriatrics. J Gerontol A Biol Sci Med Sci. 2004;59(11):1132-52.

12. Cavalini BA, Ferreira MCG, Ferreira MA. Política Nacional do Idoso e sua implementação na assistência de enfermagem. Raízes Rumos. 2014;2(1):3-11.

13. Martins L. Prevenção de doenças e promoção de saúde na terceira idade [Internet]. Rio de Janeiro: Sociedade Brasileira de Geriatria e Gerontologia; 2016 [acesso 2016 Set 06]. Disponível em: http://sbgg.org. br/prevencao-de-doencas-e-promocao-da-saude-naterceira-idade/

14. Instituto Brasileiro de Geografia e Estatística. Censo 2010: distribuição da população por sexo, segundo os grupos de idade de Mogi das Cruzes (SP) [Internet]. Brasília: IBGE; 2010 [acesso em 2013 Maio 04]. Disponível em: http://www.censo2010.ibge.gov.br/ sinopse/webservice/frm_piramide.php? codigo $=35306$ $0 \&$ corhomem $=3 \mathrm{~d} 4590 \&$ cormulher $=9 \mathrm{cdbfc}$.

15. Bezerra C. Número de médicos geriatras é insuficiente no Alto Tietê. Diário do Alto Tietê, São Paulo; ed. 2378; 2012 Out 23.

16. Oliveira JGD Neto, Carvalho DA, Sá GGDM, Monteiro MM, Lopes KDCL, et al. Pressão Arterial e Perfil Socioeconômico de Idosos Atendidos na Estratégia Saúde da Família de Floriano-Piauí. Rev de Saúde Públ Santa Cat. 2014;7(2):17-28.

17. Santos Zepka, AP, Caetano TL. A urgência no atendimento ao idoso: estudo de caso. Rev Univap. 2015;21(37):80-8.

18. Oliveira TC, Medeiros WR, Lima KC. Diferenciais sócio-demográficos da mortalidade de idosos em idades precoces e longevas. Rev Baiana Saúde Pública. 2015;39(2):249-61.

19. Virtuoso JF, Mazo GZ, Menezes EC, Cardoso ASA, Dias RG, Balbé GP. Perfil de morbidade referida e padrão de acesso a serviços de saúde por idosos praticantes de atividade física. Ciênc Saúde Coletiva. 2012;17(1):23-31.
20. Scheffer M. Demografia médica no Brasil. São Paulo: Conselho Regional de Medicina do Estado de São Paulo; 2013.

21. Sociedade Brasileira de Geriatria e Gerontologia. Folder Geriatria [acesso em 2014 Jun 20]. Disponível em: www.sbgg.org.br/publico/pdf/folder_geriatria.pdf

22. Cintra FA, Guariento ME, Miyasaki LA. Adesão medicamentosa em seguimento ambulatorial. Ciênc Saúde Coletiva. 2010;15(Supl 3):3507-15.

23. Wajngarten M, Rodrigues AG. Coração no idoso. In: Antonio Carlos Lopes, organizador. Sistema de Educação Médica Continuada à Distância - PROCLIM - Programa de Atualização em Clínica Médica. $1^{\mathrm{a}}$ ed. Porto Alegre: Artmed/Panamericana Editora; 2008. p. 9-46.

24. Manso MEG, Biffi ECA, Gerardi TG. Prescrição inadequada de medicamentos a idosos portadores de doenças crônicas em um plano de saúde no município de São Paulo, Brasil. Rev Bras Geriatr Gerontol. 2015;18(1):151-64.

25. Souza EA, Scochi NJ. Atenção ambulatorial à saúde do idoso: estudo de demanda e oferta no município de Cascavel/PR. In: IV Seminário Nacional Estado e Políticas Públicas. 2009 [acesso em 2012 Fev 21]. Disponível em: http://cacphp.unioeste.br/projetos/ gpps/midia/seminario4/trabcompletos_estado_lutas sociais_e_politicas_publicas/Trabcompleto_atencao ambulatorial_idoso.pdf.

26. Santos CTBD, Andrade LOMD, Silva MJ, Sousa MFD. Course of the elderly in health care network: a link to be built. Physis (Rio de J). 2016;26(1):45-62.

Endereço para Correspondência:

Angela Alves Correia de Souza

Rua Monte Negro, 73

Bairro: Jardim Santo Antônio

CEP 06126-030 - Osasco - SP - Brasil

E-mail: angela.aacs@yahoo.com.br 